\title{
Solid State Dynamics Visualized by Environmental EM
}

\section{Jakob Wagner}

DTU Nanolab, Technical University of Denmark, Kgs. Lyngby, Hovedstaden, Denmark

A good understanding of the dynamics and formation mechanisms of low-dimensional nanostructures from bottom-up processes is of great importance in order to exploit the controllability of the nanostructures and their applications in photovoltaics, electronics, sensors, energy related materials etc. on an industrial scale.

In situ environmental electron microscopy allows for monitoring gas and heat induced dynamics at the nanoscale in real time. Examples of such reactions include growth of 2D materials (graphene)[1], pseudo 1D materials (carbon nanotubes)[2] and semiconductor oxides such as $\mathrm{CuO}$ [3].

The high spatial resolution accessible in electron microscopy combined with spectroscopic capabilities enables fundamental insights during the dynamical processes of the nanostructured materials directly linked to growth parameters such as atmosphere composition and temperature. In the recent years, highspeed cameras and sensitive detectors have contributed to an increased temporal resolution in electron microscopy.

In situ single wall carbon nanotube (CNT) formation by means of environmental electron microscopy is directly comparable to ex situ CVD growth in terms of temperature of pressure. In order to shed light on the conditions under formation of the initial carbon cap and continuous growth of CNTs, the direct monitoring of the catalyst and CNT is essential [3].

Topology of the seed particle support, the state of the catalytic particle, and carbon source play a crucial role in the growth dynamics. Observing the initial solid carbon formation on seed particles at the atomic scale reveals the conditions leading to encapsulation of the catalyst particles (no carbon nanotube growth) and a carbon layer lift-off resulting in nanotube growth, respectively. Figure 1 shows stills from a movie recorded under formation of catalytic seed particles and subsequent SWCNT growth.

Anisotropic oxidation of metals can lead to controlled formation of nanostructures. Figure 2 shows $\mathrm{CuO}$ nanowires formed by oxidation of metallic copper in situ in the electron microscope. The geometry of the resulting structures depends strongly on the partial pressure of oxygen during the heat treatment. Low pressure results in 2-dimensional copper oxidation in contrast to the 1-dimensional $\mathrm{CuO}$ nanowires forming at higher pressures. Monitoring the growth processes directly gives valuable insight into the kinetics of the oxidation mechanism and thereby serve as platform for the models used for controlled tailor-made nanostructures by simple oxidation.

Post-growth treatment of the oxide nanowires at elevated temperature at reduced partial pressure of oxygen results in dissolution of the formed nanowires [4]. The direct observation of oxidation and disintegration reveals the nature of the facet dependent reactions.

Solid state dynamics at the nanoscale directly visualized by means of controlled atmosphere electron microscopy is a key factor in the understanding of the formation mechanisms and thereby a stepping stone towards the controlled synthesis of tailored materials. 


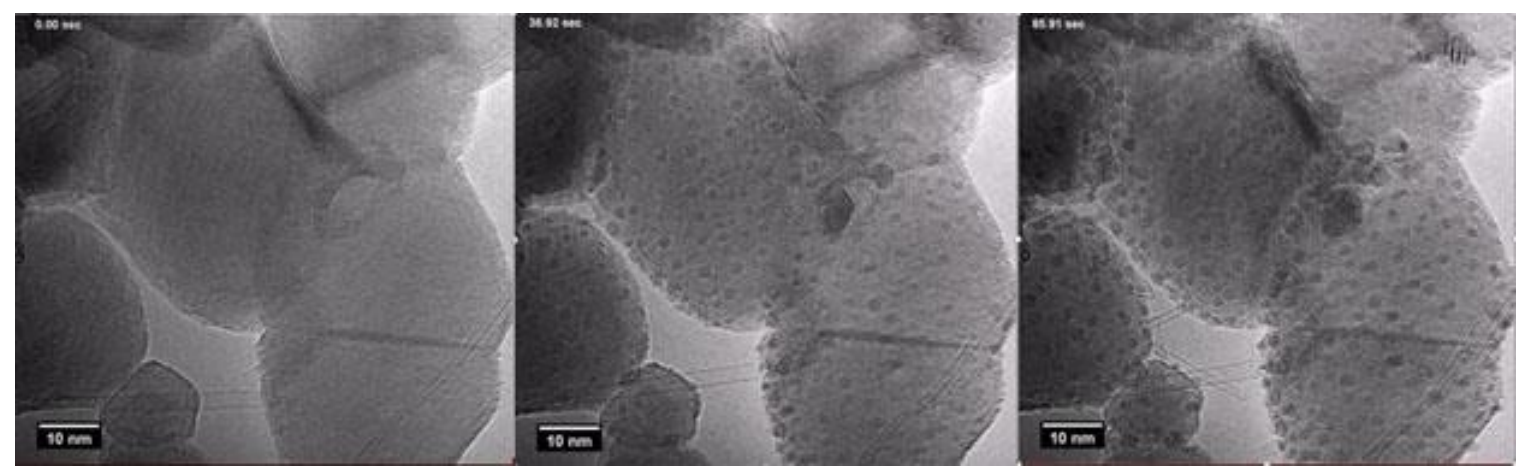

Figure 1. Formation of catalytic seed particles (Co) on a $\mathrm{MgO}$ support followed by CNT growth in $0.1 \mathrm{~Pa}$ $\mathrm{EtOH}$ at $700^{\circ} \mathrm{C}$

\section{Growth regime Transition regime} $700 \mathrm{~Pa}$
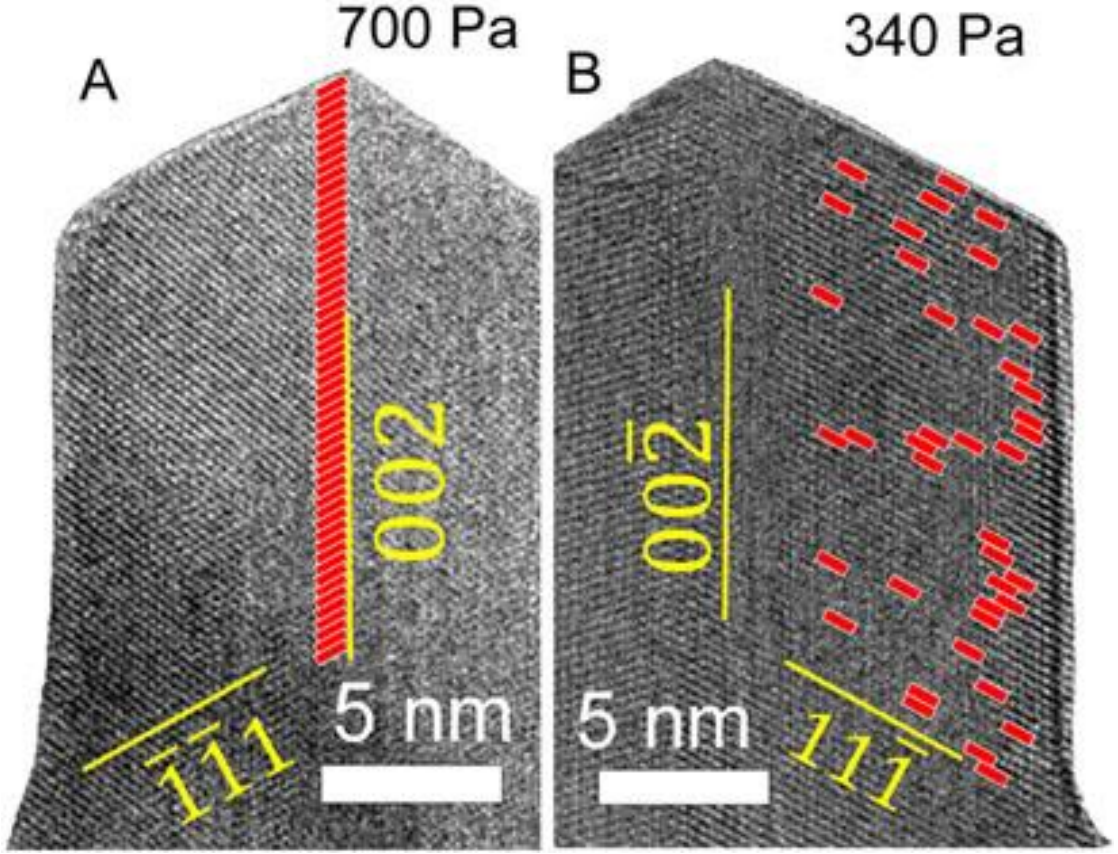

Figure 2. Oxidation of copper into a high aspect ratio $\mathrm{CuO}$ nanowire monitored by means of in situ environmental transmission electron microscopy. The layer-by-layer growth of $\mathrm{CuO}$ is heavily dependent of the vapor pressure of oxygen in the microscope. Figure adapted from [5].

\section{References}

[1] J. Kling et al. Carbon 99, 261 (2016)

[2] M. He et al., Scientific Reports 3, 1460 (2013)

[3] S. Rackauskas et al., Nano Letters 14, 5810 (2014)

[4] L. Zhang et al. ACS Nano 11, 4483 (2017)

[5] S. Rackauskas et al., Scientific Reports 7, 12310 (2017) 